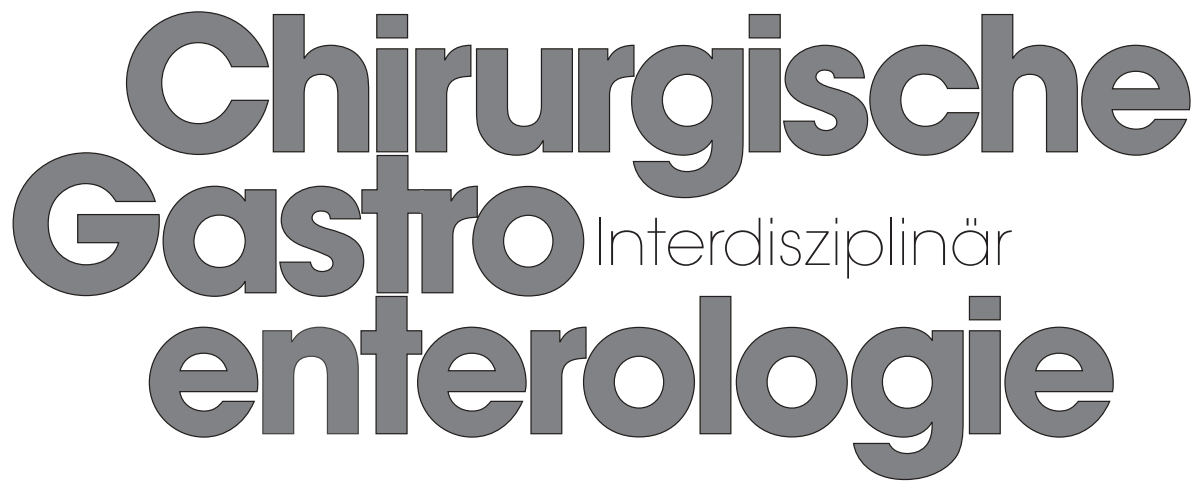

Hauptthema

J. Horn, München (Hrsg.)

\title{
Diagnostische Schritte zur Krankheitsabklärung - rational und ökonomisch
}

Eingeladene Beiträge

Interdisziplinäres Gespräch

Weitere Rubriken

Originalarbeiten

Innovationen

Buchbesprechungen

Tagungen und Kongresse 


\section{Chirurgische enterologie}

\section{Hauptschriftleitung}

R. Bittner, Stuttgart

\section{Schriftleitung Viszeralchirurgie}

E. Klar, Heidelberg

\section{Schriftleitung Gastroenterologie}

J. Mössner, Leipzig

\section{Assistenz Schriftleitung}

C.-G. Schmedt, Stuttgart

\section{Fachschriftleiter}

Viszeralchirurgie

J. R. Izbicki, Hamburg

H. Lippert, Magdeburg

H. Säuberli, Baden

R. Schiessel, Wien

Gastroenterologie

H. Bosseckert, Jena

W. G. Zoller, Stuttgart

Minimal Invasive Chirurgie

H. P. Bruch, Lübeck

I. Gastinger, Cottbus

Transplantation

C. E. Broelsch, Essen

J. Hauss, Leipzig

\section{Berufspolitik}

K.-J. Paquet, Hannover

B. Ulrich, Düsseldorf

Chirurgische Onkologie

M. W. Büchler, Bern

K. Glaser, Wien

Band 16, Heft 1, März 2000

\section{Wissenschaftlicher Beirat}

Viszeralchirurgie

J. Lange, St. Gallen

H. B. Reith, Würzburg

H.-D. Saeger, Dresden

Gastroenterologie

W. E. Fleig, Halle

M. Fried, Zürich

E. G. Hahn, Erlangen/Nürnberg

J. Schölmerich, Regensburg

Minimal Invasive Chirurgie

F. Köckerling, Hannover

C. Meyer, Straßburg

R. Schlumf, Aarau

K. Schönleben, Ludwigshafen

G. Szinicz, Bregenz

W. Wayand, Linz

Berufspolitik

M. Betzler, Essen

K. Junghanns, Ludwigsburg

Chirurgische Onkologie

W. Hohenberger, Erlangen/Nürnberg

Onkologie

S. von Hegewisch-Becker, Hamburg

R. Porschen, Tübingen

W.-H. Schmiegel, Bochum

\section{Internationaler Beirat}

J. Gallinger, Moskau

J. M. Henderson, Cleveland

I. Ihse, Lund

C. W. Imrie, Glasgow

S. L. Jensen, Arhus

K. Maruyama, Tokyo

W. C. Meyers, Worcester
Endoskopie

S. Liebe, Rostock

P. Malfertheiner, Magdeburg

H. Neuhaus, Düsseldorf

J. F. Riemann, Ludwigshafen

Perioperatives Management

K. W. Jauch, Regensburg

H. Wiedeck, Ulm

D. H. Wittmann, Wisconsin

K. Hell, Basel

Radiologie

M. Langer, Freiburg

Pathologie

P. Hermanek, Erlangen/Nürnberg

C. Wittekind, Leipzig

Klinische Molekularbiologie

H. Fries, Bern

Innovation, Aktuelle Trends,

Kongreßberichte

E. Klar, Heidelberg

J. Mössner, Leipzig

W. G. Zoller, Stuttgart

Buchbesprechungen

K.-J. Paquet, Hannover

E. Moreno-Gonzáles, Madrid

F. M. Penninckx, Leuven

A. Peracchia, Mailand

L. F. Rikkers, Madison

J. Rodés, Barcelona

J. Terblanche, Kapstadt

A. L. Warshaw, Boston

S. Karger $\cdot$ Medical and Scientific Publishers $\cdot$ Basel $\cdot$ Freiburg $\cdot$ Paris $\cdot$ London $\cdot$ New York $\cdot$ New Delhi $\cdot$ Bangkok $\cdot$ Singapore $\cdot$ Tokyo $\cdot$ Sydney

Die Zeitschrift erscheint vierteljährlich: pro Jahr erscheint 1 Band zu je 4 Heften. Bezugspreis für Jahrgang 16, 2000: DEM 230.- / SFr 179,-- einschließlich MWSt., zuzüglich Postgebühren. Der Abonnementpreis ist im voraus zahlbar. Das Abonnement der Zeitschrift läuft weiter, wenn es nicht spätestens 4 Wochen vor Abschluß eines Bandes abbestellt wird.

Abonnementbestellungen können bei jeder Buchhandlung oder direkt beim Verlag aufgegeben werden:

Deutschland:

S. Karger $\mathrm{GmbH}$

Lörracher Str. 16 a

D-79115 Freiburg

Tel. +49761452070

Fax +497614520714

E-mail Information@Karger.de Fax +41613061234

E-mail Karger@Karger.ch
Anzeigen: S. Karger

Verlag für Medizin und Naturwissenschaften $\mathrm{GmbH}$

Lörracher Str. 16 a. D-79115 Freiburg, Tel. +49 761452070

Gültig ist die Preisliste Nr. 7 vom 1. Januar 1999

Für den Inhalt außerhalb des redaktionellen Teils (insbesondere Anzeigen, Industrieinformationen, Pressezitate und Kongreßinformationen) übernehmen Schriftleitung, Beirat und Verlag keine Gewähr.

Eine Markenbezeichnung kann warenzeichenrechtlich geschützt sein, auch wenn bei ihrer Verwendung in dieser Zeitschrift das Zeichen ${ }^{\circledR}$ oder ein anderer Hinweis auf etwa bestehende Schutzrechte fehlen sollte. Für Satzfehler, insbesondere bei Dosierungsangaben, wird keine Gewähr übernommen.

Die Zeitschrift sowie alle in ihr enthaltenen einzelnen Beiträge und Abbildungen sind urheberrechtlich geschützt. Jede Verwertung, die nicht ausdrücklich vom Urheberrechtsgesetz zu- gelassen ist, bedarf der vorherigen Zustimmung des Verlags. Das gilt insbesondere für Vervielfältigung, Bearbeitungen, Übersetzungen, Mikroverfilmungen und die Einspeicherung und Verarbeitung in elektronischen Systemen.

C C Copyright 2000 by S. Karger

Verlag für Medizin und Naturwissenschaften $\mathrm{GmbH}$ Lörracher Straße 16 a, D-79115 Freiburg

Verlagsleitung und presserechtlich verantwortlich: Sibylle Hopf

Redaktionsassistenz: Dr. Hannelore Tenckhoff

Anzeigenleitung: Susanne Meister

Produktionsleitung: Georg Brunner

Herstellung: druckhaus köthen $\mathrm{GmbH}$

Friedrichstr. 11/12, D-06366 Köthen/Anhalt

ISBN 3-8055-7051-1

\section{KARGER}

Fax +497614520714

E-mail Informatio

www.karger.con (c) 2000 S. Karger GmbH, Freiburg

Home Page der Zeitschrift:

www.karger.com/journals/cga
Bibliographische Dienste:

EMBASE / Excerpta Medica

Reference Update 
Laudatio

5 Sechzehn Jahre Schriftleitung der CHIRURGISCHEN GASTROENTEROLOGIE

Horn, J. (München)

Editorials

7 CHIRURGISCHE GASTROENTEROLOGIE im Aufbruch Bittner, R. (Stuttgart)

8 Diagnostische Schritte zur Krankheitsabklärung - rational und ökonomisch

Horn, J. (München)

Hauptthema

10 Rationale und rationelle Diagnostik der Peritonitis Billing, A.; Helmberger, T. (München)

18 Rationelle Diagnostik beim Leitsymptom Ileus

Langenscheidt, P.; Gohl, D. (Homburg)

28 Leitsymptom Erbrechen: Diagnostik und Therapie aus chirurgischer Sicht Stöhr, G. (Göttingen)

32 Diagnostische Schritte zur Krankheitsabklärung bei Ikterus Heise, J. W.; Röher, H.-D. (Düsseldorf)

41 Rationelle Diagnostik zur Abklärung der gastrointestinalen Blutung

Bumm, R. (München)

46 Abklärung chirurgischer Ursachen einer chronischen Anämie

Schmidt-Matthiesen, A. (Frankfurt/Main)

52 Rezidivierende Durchfälle: Klinisch orientierte differentialdiagnostische Abklärung Stadelmeier, U.; Hohenberger, W. (Erlangen)
Laudation

516 Years Editorship of CHIRURGISCHE GASTROENTEROLOGIE

Horn, J. (München)

Editorials

7 CHIRURGISCHE GASTROENTEROLOGIE in Departure Bittner, R. (Stuttgart)

8 Diagnostic Pathways for Disease Clarification - Rational and Efficient

Horn, J. (München)

Main Theme

10 Rational and Efficient Diagnostics of Peritonitis Billing, A.; Helmberger, T. (München)

18 Rational Diagnostics: Intestinal Obstruction as the Principal Sign

Langenscheidt, P.; Gohl, D. (Homburg)

28 Vomiting as the Principal Sign: Diagnostics and Therapy from a Surgical Viewpoint

Stöhr, G. (Göttingen)

32 Diagnostic Procedures to Clarify Jaundice Heise, J. W.; Röher, H.-D. (Düsseldorf)

41 Rational Diagnostics for Clarification of Gastrointestinal Bleedings

Bumm, R. (München)

46 Clarification of Surgical Reasons for Chronic Anemia

Schmidt-Matthiesen, A. (Frankfurt/Main)

52 Recurrent Diarrhoea: Clinically Oriented Differential Diagnostic Clarification Stadelmeier, U.; Hohenberger, W. (Erlangen)

\section{KARGER}

Fax +497614520714

E-mail Informatio

www.karger.com (c) 2000 S. Karger GmbH, Freiburg

Artikel (Volltext) und Inhaltsverzeichnisse sowie das vorläufige Inhaltsverzeichnis des nächsten Heftes: www.karger.com/journals/cga/cga_bk.htm 
61 Diagnostische Schritte zur Krankheitsabklärung beim Leitsymptom Gewichtsabnahme

Schröder, J.; Wesner, F.; Kremer, B. (Kiel)

65 Diagnostik des chronischen Unterbauchschmerzes Nagel, M.; Wehrmann, U.; Ringelband, B. (Dresden)

Interdisziplinäres Gespräch

70 Rationale Diagnostik gastrointestinaler Erkrankungen Zoller, W. G. (Stuttgart)

Originalarbeiten

75 Eine neue, zentralisierte und gestraffte Arbeitsstruktur macht die Chirurgie effizienter

Hedenbro, J. L.; Rydén, C. I.; Staël von Holstein, C.; Ihse, I. (LundLandskrona)

81 Endoskopische Therapie für Subtypen des Magenkarzinoms im Frïhstadium

Shimao, H.; Hiki, Y.; Morise, M.; Kikuchi, S.; Kobayashi, N.; Sakakibara, Y.; Kakita, A.; Tanabe, S.; Saigenji, K. (Sagamihara); Tsutsumi, O. (Braunschweig)

85 Innovationen

87 Buchbesprechungen

89 Tagungen und Kongresse

2 Impressum

U2 Hinweise für Autoren
61 Diagnostic Procedures in Patients with the Principal Sign of Weight Loss

Schröder, J.; Wesner, F.; Kremer, B. (Kiel)

65 Diagnostics of Chronic Lower Abdominal Pain Nagel, M.; Wehrmann, U.; Ringelband, B. (Dresden)

Interdisciplinary Discussion

70 Rational Diagnostics of Gastrointestinal Diseases Zoller, W. G. (Stuttgart)

Original Articles

75 Proper Structure of a Surgical Department is Important for Its Efficiency

Hedenbro, J. L.; Rydén, C. I.; Staël von Holstein, C.; Ihse, I. (LundLandskrona)

81 Endoscopic Treatment for Subtypes of Early Gastric Cancer

Shimao, H.; Hiki, Y.; Morise, M.; Kikuchi, S.; Kobayashi, N.; Sakakibara, Y.; Kakita, A.; Tanabe, S.; Saigenji, K. (Sagamihara); Tsutsumi, O. (Braunschweig)

\section{Innovations \\ 87 Book Reviews \\ 89 Meetings and Conferences}

2 Imprint

U3 Guidelines for Authors

\section{KARGER}

Fax +497614520714

E-mail Information@Karger.de

www.karger.com
(C) 2000 S. Karger GmbH, Freiburg 